\title{
Fuzzy Neural Network Control System Design for Solar Water Heater
}

\author{
Zhi-Da ZHU a, , Li ZENG ${ }^{\text {b }}$, Fan ZHANG ${ }^{c}$ \\ College of Mechanical Engineering Yangzhou University, Yangzhou 225127, China \\ azdzhu@yzu.edu.cn, blizengcf@163.com, c fzhang@yzu.edu.cn
}

Keywords: Fuzzy neural network, Solar, Water heaters, MCU, ANFIS.

\begin{abstract}
There are more and more applications of Solar water heater in urban and rural, the research and development of advanced Solar water heater control system becomes more and more important, to improve the working performance and convenience of solar water heater. According to the requirements of the solar water heater control, a new control system of solar water heater is put forward.
\end{abstract}

\section{Introduction}

With the growing of global population, the demand for energy is getting higher and higher. But the main energy resources, such as petroleum and coal, cannot adapt to the modern requirement of low carbon energy and no-pollution energy. So the solar energy, as a new type of energy, has been paid more and more attention to, which has advantages of safety, environmental protection, energysaving and unlimited reserves [1-5]. Solar water heater is a kind of equipment which can convert solar energy into heat energy. It includes some vacuum glass tubes for collecting heat, a tank for storing water, brackets and other accessories.

Now there are many kinds of solar water heater on the market, but most of solar water heater cannot adopt automatic requirements for solar water heater. The solar water heater control systems in the market can be divided into the semi-automatic controller, the full-automatic controller and the whole intelligent controller. Semi-auto- matic controller can get power supply from battery pack or $220 \mathrm{~V}$ city power grid, which has single function, low price, and some simple detection function, such as water level and water temperature display, and alarm of lack water or full water. The operations of opening valve and supplying water or closing valve without water are manual; users cannot control the water heater by it. So it actually is only a display instrument, and is called Semiautomatic controller. Full- automatic controller is composed of a host, some sensors and electromagnetic valves, which has the functions of semi-automatic controller and the functions as manual water supplying, automatic water supplying (water supplying at the set time, or when water shortage, or when water pressure in tank is too low) and heat preservation of conduit, etc. Some of these controllers have the functions as water supplying with boosting and anti-freezing, which can meet the climate and usage of different regions. The structure of intelligent controller is difference from full automatic controller which adds the electric auxiliary heater. It has not only the functions of full automatic controller, but also the functions as intelligent heating, heating at the set time, smart water supplying, leakage protection, time displaying, memory when electricity is broken. So it can not only ensure the full automatic water supplying, but also the enough hot water. Some of these controllers can maintain the water temperature and water level.

Most of the solar water heater intelligent control systems use the micro controller chip, and have just a single function and some complex operations, so they are inconvenience of control and poor of anti-interference ability. Especially there is no good control on the water temperature. even if it has the auxiliary heating function, the insufficient heating or burning still often appear. These would not only cause very big potentially dangerous, but also waste a large amount of electrical energy [6-12]. As an important branch in the field of intelligent control, fuzzy control and artificial neural network (ANN) represents the future development directions of the control technology. And the fuzzy neural network is a neural network and fuzzy logic combined product based on the function complementary between them which provides the conditions for their common control [13-17]. The fuzzy neural network combines the advantages of fuzzy control and artificial neural 
network, so this paper based on fuzzy neural network to design a control system of solar water heater.

\section{Design of Fuzzy Neural Network}

In this paper, the temperature error, error change rate as input of the network, calculate the parameters of the membership function and the corresponding fuzzy rules by using the learning ability, so as to obtain an output that is electrically heated start time. The whole fuzzy neural network structure is shown in figure 1.

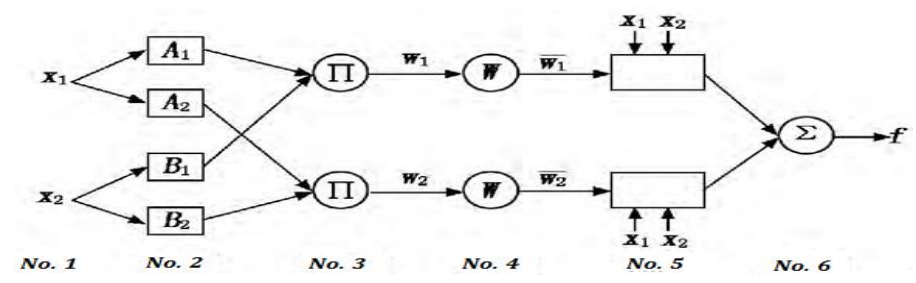

Fig.1 Structure of Fuzzy Neural Network

The input of this fuzzy neural network is:

$$
\begin{aligned}
& x_{1}=e(t) \\
& x_{2}=\Delta e(t)=\frac{[e(t+\Delta t)-e(t)]}{\Delta t}
\end{aligned}
$$

In the $\mathrm{Eq}, \mathrm{e}(\mathrm{t})$ is the current temperature, $\Delta \mathrm{e}(\mathrm{t})$ is the rate of change of temperature. Assuming that each input has $\mathrm{n}$ membership, the fuzzy neural network in each layer of the relationship between input and output is as follows [18].

(1) The first layer is the input layer. The $\mathrm{x} 1$ and $\mathrm{x} 2$ respectively represent the temperature error and the rate of error change. Each input variable transfers to the next layer without any treatment, the input of this layer and the output unit respectively are:

$$
\begin{array}{ll}
\boldsymbol{I}_{i}^{(1)}=x_{i} & i=1,2 . \\
\boldsymbol{O}_{i j}^{(1)}=\boldsymbol{I}_{i}^{(1)} & i=1,2 . ; j=1,2, \cdots, n .
\end{array}
$$

(2) The second layer is a layer of language. Each note represents a linguistic variable; using gauss function as membership function, blur the input variables, and calculate the fuzzy membership of the input variables. The input unit and output unit of this layer respectively:

$$
\begin{aligned}
& I_{i j}^{(2)}=-\frac{\left(O_{i j}^{(1)}-c_{i j}\right)}{\sigma_{i j}^{2}} \quad i=1,2 ; j=1,2, \cdots, n . \\
& O_{i j}^{(2)}=\mu\left(A_{i j}\right)=\exp \left(I_{i j}^{(2)}\right) i=1,2 . ; j=1,2, \cdots, n .
\end{aligned}
$$

In the Eq, oij and cij respectively represent the width and center of the Gauss function.

(3) The third layer is the rule layer. Each node represents a fuzzy rule, and one correspondence, which can obtain the input unit and output unit of this layer respectively are:

$$
\begin{aligned}
& \boldsymbol{I}_{j(n+l)}^{(3)}=\boldsymbol{O}_{1 j}^{(2)} \times \boldsymbol{O}_{1 l}^{(2)} \quad j=1,2, \cdots, n ; l=1,2, \cdots, n . \\
& \boldsymbol{O}_{i}^{(3)}=\omega_{i}=\boldsymbol{I}_{i}^{(3)} \quad i=1,2, \cdots, m .
\end{aligned}
$$

In the Eq, $\mathrm{m}=\mathrm{n} 2$. The layer can be any AND operator with $\mathrm{T}$ paradigm, the excitation intensity of each fuzzy rule can output by operation, which is $\omega \mathrm{i}$ in the Eq.

(4) The fourth layer is one layer. Every note of this layer are denoted by W, by normalization of relevance grade of each rules, to calculate the ratio of incentive intensity at i point and incentive intensity of all rules. The input unit and output unit respectively are: 


$$
\begin{aligned}
& \boldsymbol{I}_{i}^{(3)}=\boldsymbol{O}_{i}^{(3)}=\omega_{i} \quad i=1,2, \cdots, m . \\
& \boldsymbol{O}_{i}^{(3)}=\bar{\omega}_{i}=\frac{\omega_{i}}{\sum_{i} \omega_{i}} \quad i=1,2, \cdots, m .
\end{aligned}
$$

(5) The fifth layer is the rule of output layer. This layer is used to calculate the output of each fuzzy rule, which can obtain the input unit and output unit respectively are:

$$
\begin{aligned}
& I_{i}^{(5)}=O_{i}^{(5)}=\bar{\omega}_{i} \quad i=1,2, \cdots, m . \\
& O_{i}^{(5)}=\bar{\omega}_{i} f_{i}=\bar{\omega}_{i}\left(p_{i} x_{i}+q_{i} x_{2}+r_{i}\right) \quad i=1,2, \cdots, m .
\end{aligned}
$$

In the Eq, pi, qi, ri are conclusion parameters of the linear system. And fi is a linear combination value of the conclusion parameter.

(6) The sixth layer is the overall output level. The unit of the layer is a fixed node, the sum of all input signal as the output. The input unit and output unit of the layer are respectively:

$$
\begin{aligned}
& \boldsymbol{I}_{i}^{(6)}=\boldsymbol{O}_{i}^{(5)}=\bar{\omega}_{i} f_{i} \quad i=1,2, \cdots, m . \\
& \boldsymbol{O}_{i}^{(6)}=\sum \bar{\omega}_{i} f_{i}=\frac{\sum_{i} \omega_{i} f_{i}}{\sum_{i} \omega_{i}} \quad i=1,2, \cdots, m .
\end{aligned}
$$

For this fuzzy neural network, after determine the initial membership functions, the total output of the system is linear combination of conclusion parameters. For many parameters used in the network, so a hybrid learning algorithm is used, which increases the least square method on the basis of gradient descent method[19].

Objective function defined is:

$$
E=\frac{1}{2}(D-Y)^{2}=\frac{1}{2} e^{2}
$$

In the Eq, D is the desired output, which is teacher signal; $Y$ is the actual output of the system.

In the mixed learning process, when learning to the fifth layer, the conclusion parameters are pi, qi, ri by least square method. Through reverse learning of maximum gradient method, each parameter correction value of membership function by a layer of calculation is:

$$
\begin{gathered}
\Delta c_{i j}=-\frac{\partial E}{\partial c_{i j}}=-\frac{\partial E}{\partial \boldsymbol{I}_{j}^{(2)}} \frac{\partial \boldsymbol{I}_{j}^{(2)}}{\partial c_{i j}}=\delta_{j}^{2} \frac{2\left(y_{j}^{1}-c_{i j}\right)^{2}}{\sigma_{i j}^{2}} \\
\Delta \sigma_{i j}=-\frac{\partial E}{\partial \sigma_{i j}}=-\frac{\partial E}{\partial \boldsymbol{I}_{j}^{(2)}} \frac{\partial \boldsymbol{I}_{j}^{(2)}}{\partial \sigma_{i j}}=\delta_{j}^{2} \frac{2\left(y_{j}^{1}-c_{i j}\right)^{2}}{\sigma_{i j}^{3}}
\end{gathered}
$$

Among them, $i=1,2 ; j=1,2, \ldots, 5$; is the local gradient layer, is the concrete actual output.

The paper identifies the parameters of conclusion section by way of off-line and collects a large number of dates of temperature and irradiance which we need with the help of the equipment data acquisition instrument of a solar energy Ltd test experiments. Import the data into MATLAB, through ANFIS simulation toolbox to simulation training [20], according to the above algorithm and the related parameters. In order to improve the training efficiency, the number of network training set at 40 times, and the error curve of this simulation is shown in Figure 2.

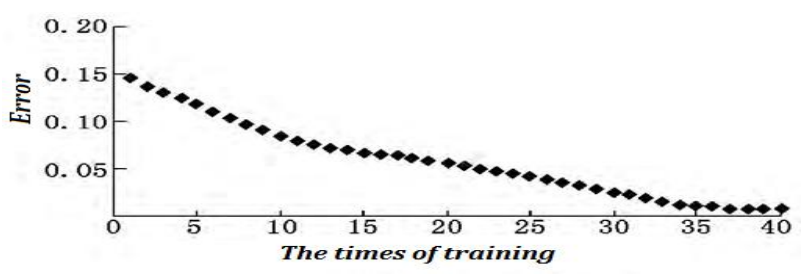

Fig.2. Simulation Curves of Fuzzy Neural Network 
As can be seen from Figure 2, after 40 rounds of training, at this moment, the network error is 0.0068107. So when training to 40 times, the network performance has been reached. Then, the trained ANFIS neural network can be used as a method written into MCU to control.

\section{Hardware Design}

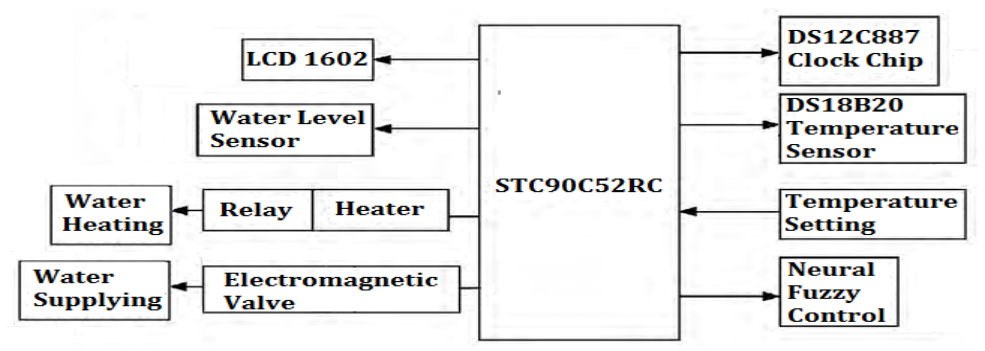

Fig.3. Block Diagram of the Control Hardware

In the aspect of hardware, the fuzzy neural network control system of solar water heater is composed of single chip microcomputer, LCD, clock chip, temperature sensors, water level sensors, auxiliary electric heater, electromag- netic valves and so on. The control system gets signals of solar water heater working state through one or several reliable sensors detecting and processing, and is operated by a single chip computer, which uses the base established for control rules to simulates the working state of the human brain, then controls the work of solar water heater. It uses the lowcost MCU micro controller and software based on the fuzzy inference algorithm whose parameters are gotten from software training and learning to replace the high cost special fuzzy microcontroller. The LCD module is used to display the control rules setting and the actual working conditions of the solar water heater control system, and operate the solar water heater. The hardware diagram is shown in Figure 3.

(1) The Clock Chip DS12C887

The system selects DS12C887 as the clock chip to display real-time clock, and can automatically save the settings during power failure, as well as do not break the operation of the clock. The interface of clock chip is shown in Figure 4.

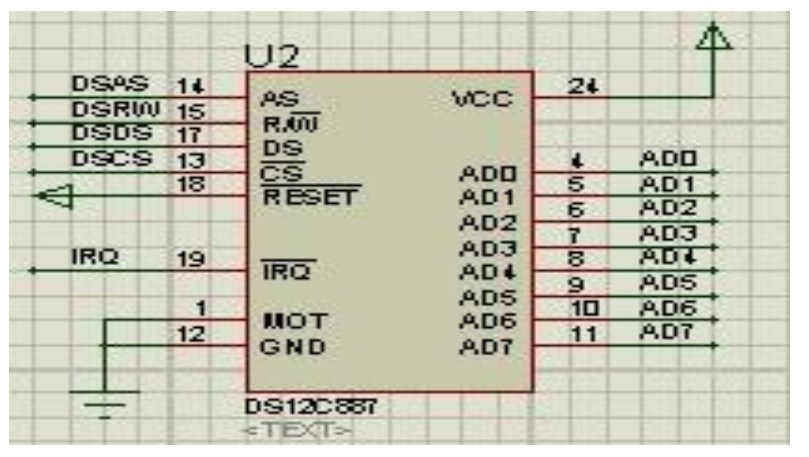

Fig.4. the Interface of Clock Chip

(2) The Temperature Detecting Circuit

To detect the temperature of the water tank is an important part of control system, the key of which is getting the accurate temperature signal, and then obtaining accurate control effect and achieving the purpose of saving electricity with the software for controlling the auxiliary heating. In order to reduce the peripheral circuit and increases the reliability, the paper adopts 1-wire digital temperature sensor DS18b20 produced by American Dallas company, which has small volume, high resolution, high speed, 9 bit digital form to reflect the temperature of the device, there is no need for A/D converter. Its working circuit o is shown in Figure 5: 


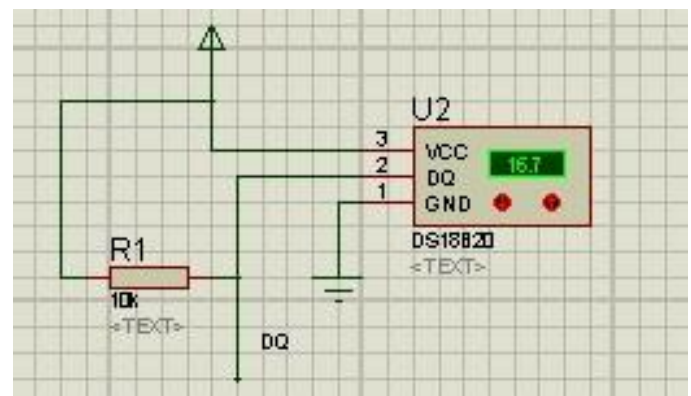

Fig.5. The working circuit diagram of DS18B20

DS18B20 needs one signal line connected to SCM, which takes only 1 bit I/O interface of SCM. and this 1 bit I/O interface can connect multiple DS18B20s through interface sharing, so it can realize single point or multi-point temperature detection. DS18B20 sensor has high precision and good interchangeability. it can directly encodes the temperature data, so the temperature data can be transmitted by only one single cable, which brings not only convenient communication, but also far transmission distance and good anti interference. Compared with the multi-point temperature measurement system which uses the traditional temperature sensors, it can save a large amount of cable, and simplify the system, and cause the system expansion and maintenance very easy.

(3) The Water Level Detecting Circuit

Water level detection is also a very important part of this fuzzy control system. On the market today, most of the water level sensors used in solar water heater are segmented, which are so much variety, such as electrode sensor for water and electricity contact and float sensors using Hall effect etc al, but the value measured by segmented water level sensor brings great inconvenience to system precise controlling. Here the design idea of using pressure sensor for detecting water level is used, which converts the water level detection into the water weight detection. The sensor gets the change of pressure value, processes it with the circuit integrated in sensor and outputs the square wave with different frequency, then through the processing of software the water level in the water tank can display on LCD screen. The detection of the pressure sensor for water level is continuously variable, so we can finally get the continuous measurement of the water level. The water level sensor is shown in figure 6 .
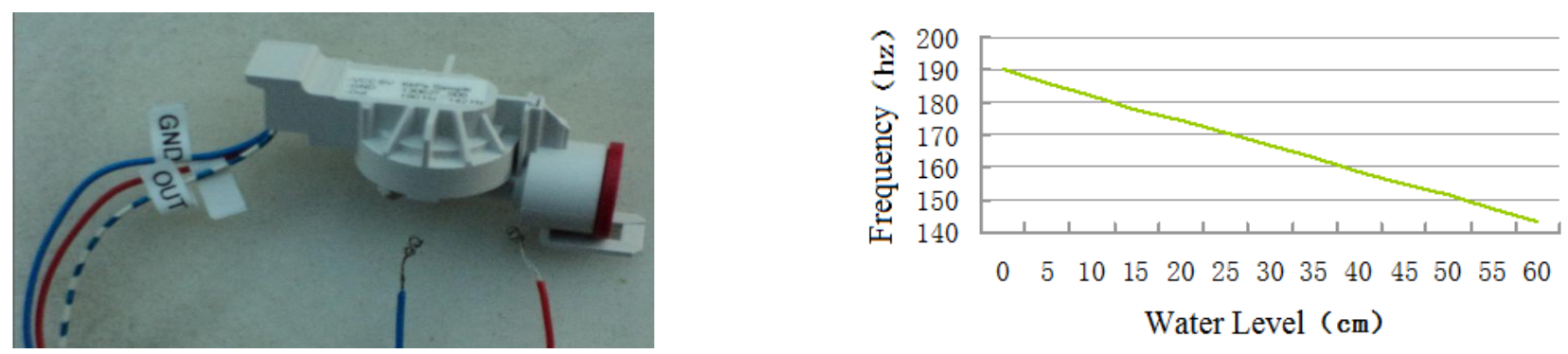

Fig.6. The Water Level Sensor

Fig.7 The Relationship Curve of Frequency and Water Level

The relationship of water level and pressure frequency from water level sensor should be linear, which is shown in Figure 7.From the curve of Figure 7, we can know that while the water level increases, the frequency is lowered. After measurement experiment, the frequency characteristic of water level can be gotten: when the water level is $0 \mathrm{~cm}$, the frequency is $44.3 \mathrm{hz}$; when the water level is $75 \mathrm{~cm}$, the frequency is $42.16 \mathrm{hz}$; when the water level is $150 \mathrm{~cm}$, the frequency is $40 \mathrm{~Hz}$; when the water level is $225 \mathrm{~cm}$, the frequency is $37.77 \mathrm{hz}$; when the water level is $300 \mathrm{~cm}$, the frequency is $35.39 \mathrm{hz}$.

(4) The Auxiliary Heating Control Circuit

The control system uses a normal open contact of the solid state relay as a switch for connecting the auxiliary electric heater. Because the heating of auxiliary heater is realized by the heating resistance wire, which is a high power AC component, and the pin of STC90C52RC single chip 
computer cannot support such a strong current, it need use the power amplifier component controlling the $220 \mathrm{~V}$ alternating current to heat the water. The electric heating control circuit is shown in Figure 8. When the light emitting diode in the solid state relay is conducting, the current flows should go through the right circuit in relay U4, then the normal open contact should start working and the auxiliary heater should be switched on to heat water. The diode D4 is used to protect the working circuit while the too large reverse current should appear. The initial control signals of the relay action or not is from Pl.0 interface pin of STC90C52RC. While the CPU sends a high-level voltage to the P1.0, the Q3 transistor begins to work, then the solid state relay is switched on and the D3 light emitting diode is lit, that means the auxiliary heater being in the working state. When the temperature is less than or equal to $3^{\circ} \mathrm{C}$, the auxiliary electric heater is automatically opened. But when the water temperature is equal to $10^{\circ} \mathrm{C}$, the auxiliary electric heater is automatically shut off.

(5) The Water Supplying Control Circuit

In addition to operate the electric heater, the solenoid valve is also used to supply the water. The water supplying control can not only ensure enough water in the tank, but also keep the water temperature not too high. When the temperature is $2^{\circ} \mathrm{C}$ higher than the storage temperature requirements, the water supplying should be opened automatically, then the water can be cooled after the water is mixed; and when the temperature is $2^{\circ} \mathrm{C}$ lower than the storage temperature requirements, the water supplying should be automatically shut off. When the temperature is equal or less than $3^{\circ} \mathrm{C}$ and the water level is lower than the water level of anti-dry burning, the water supplying should be opened automatically. After reaching the water level of anti-dry burning, the water supplying is automatically shut off and the electric heater is opened.

The control circuit of the water supply is shown in Figure 9, while the output signal from the Pl.1 interface pin of the STC90C52RC is a high-level voltage, the 2N7000 tube MOS is conducted, then the electromagnetic coils work, so that the solenoid valve is opened. In Figure 9, the green LED D6 is used, instead of solenoid valve.

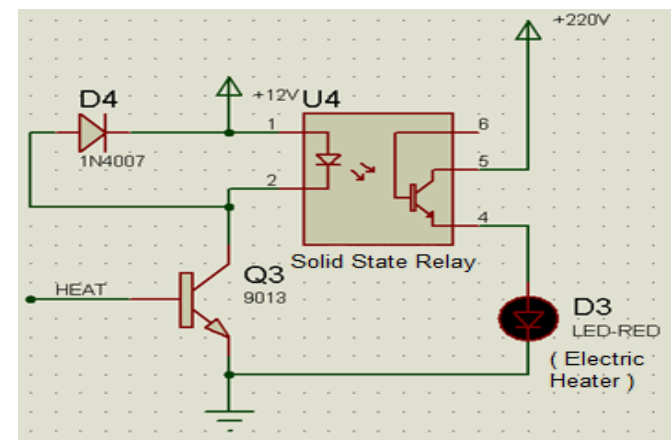

Fig.8. The working circuit diagram of DS18B20

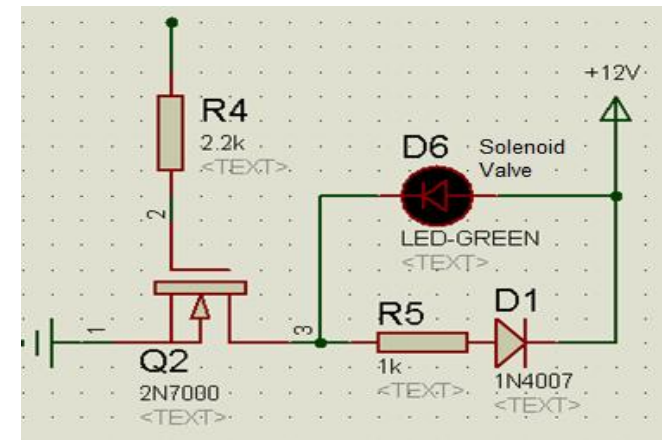

Fig.9. The working circuit diagram of DS18B20

\section{Software Design}

The control operation of the solar water heater should be done under the control of the software system. At the same time, the software can make the control function of the system more flexible and convenient, and reduce the dependence on hardware control algorithm and structure. In order to design the software program, the requirement to the control system of the water heater must be defined. the following functions should be implemented:

(a) To collect the water temperature and water level data;

(b) Through the keys to achieve manual control water supply, heat, and a variety of user set;

(c) The non-volatile RAM achieved power down protection data will be stored in the DS12C887;

(d) The data collected displayed in a timely manner through the liquid crystal;

(e) When the water level is too high or too low or the temperature is too high, give an alarm through the buzzer to remind the user operation. 


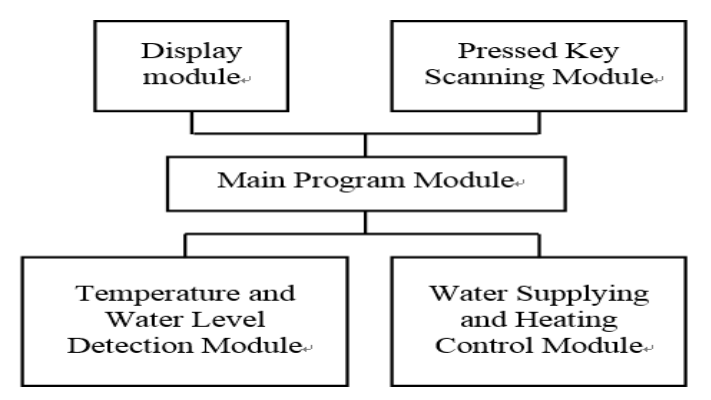

Fig.10. the Block Diagram of the Control Software

According to the above requirements, According to the above requirements, the variables that need to be measured are the error of water temperature, the rate of error change and the height of water level. The variables that need to be controlled are the volume of automatic water supplying and the time consuming of electric heating. And time, temperature and water level should be detected and displayed on the LCD in real time. The system is designed with modular design method, which includes main control program, the subprogram of water temperature and water level data collection, keyboard scanning subroutine, LCD displaying subroutine, the interrupt subprogram of DS12C887 time and alarm, early heating time calculation subroutine and so on. , as shown in figure 10 . The program is compiled with the $\mathrm{C}$ language, short and simple, fast running speed, easy programming and debugging.

(1) Main Program Module

The system main program module mainly completes the detection of temperature and water level as well as the auxiliary heating time budget and some initialization function. In the main program, the fuzzy neural network method is used to carry out the auxiliary heating in advance. Because the training of fuzzy neural network learning has been finished by off-line method, the network can be calculated directly. This saves the machine when it does not require too much.

(2) Pressed Key Scanning Module

In the push pull subroutine module needs to complete the water level setting, the water temperature settings, the appointment of water time settings, time calibration, etc. A key to the identification of the main scan to set a data register for a 0 or 1 to determine. At the same time, the software for jitters elimination method, using time delay procedures to skip the jitter, when determining whether a key is pressed, the first implementation of a greater than 10ms delay procedures to determine whether does have a button is pressed, thereby eliminating due to disoperation caused by error touch.

$\mathrm{S} 1$ is the function key, which is used to switch the operation state. Control system has two kinds of working conditions, "direct control" and "parameter modification". When the S1 is not pressed, the control system for the "direct control" state, can be directly accepted by the user to control the heating or water operation. Into the "direct control", S2 used to manual heating, S3 to manual Water Supplying, S4 to stop heating or Water Supplying; if the water level has exceeded the limit water level setting, or the water temperature has the temperature exceeds the set, "direct control" will not work.

(3) Temperature and Water Level Detection Module

The temperature detection module is used to collect the subroutine the temperature of the water in the water tank of water heater. Because this system uses the digital temperature sensor, although the peripheral circuit is little, but the programming request is also high, needs to be measured in the program the simulation quantity to be converted into the digital quantity.

(4) Water Supplying and Heating Module

Heating module in the main is the auxiliary heating electric heating, and this part is the neuro fuzzy control before we say chapters. According to the measured temperature error and error change rate to calculate the start time of the electric heating, the module of the part of the program is as follows, membership function of fuzzy rules etc. have been in the previous chapter listed here do not repeat them. 


\section{Conclusion}

In this paper, a fuzzy neural network control system is designed for solar water heater, and the following works are done to improve the control performance:

1) Because the temperature of the solar water heater is uncertain, and the mathematical model of the solar water heater cannot be established, the fuzzy neural network is used to control the temperature, which could be fitter than traditional control algorithm.

2) The fuzzy neural network controller is designed, and the fuzzy neural network control algorithm of the system is deduced. Then the simulation is done for the fuzzy neural network basing on the experimental data with the ANFIS toolbox. The simulation results show that the system has reached the performance requirements after 40 times training;

3) Based on the control algorithm, the hardware system for the solar water heater controlling is established with STC90C52RC microcontroller, which could realize the fuzzy neural network control of the water heater.

\section{Acknowledgements}

Thank the China Natural Science Foundation for this project. Item Number: 51375427, 51475409. Thank the Jiang Su Natural Science Foundation for this project. Item Number: BK20131232, BK20141277. Thank the Jiang Su Natural Science Foundation for this project. Item Number: BY2014117-08, BY2015061-04.

\section{References}

1. K.K. Chong, K.G. Chay, K.H. Chin, "Study of a solar water heater using stationary V-trough collector," Renewable Energy, Vol.39, No.1, pp.207-215, March 2012.

2. R. Kumar, R.S. Adhikari, H.P. Garg, A. Kumar, "Thermal performance of a solar pressure cooker based on evacuated tube solar collector," Applied Thermal Engineering, Vol.21, pp.16991706, 2001.

3. Kamyar Tanha, Alan S. Fung, and Rakesh Kumar, "Performance of two domestic solar water heaters with drain water heat recovery units: Simulation and experimental investigation," Applied Thermal Engineering, Vol.90, No.5, pp.444-459, November 2015.

4. Dominic B. Solpico et al., "Solar-Powered Field Server and Aerator Development for Lake Palakpakin," Journal of Advanced Computational Intelligence and Intelligent Informatics, Vol.18 No.5 , 2014, pp. 755-763

5. Dapeng Li, Shengming Liao, "An integrated approach to evaluate the performance of solar water heater in the urban environment," Energy and Buildings, Vol.69, pp.562-571, February, 2014.

6. Zhang Xianchen, "the design of single chip microcomputer controller of a practical solar water heater controller," Automation and Instrumentation, Vol.12, No.6, pp.19-21, 2005.

7. Huo Zhenyu, Ma Yongqiang, Yang Zhu, "Design of intelligent Control System for Solar Water Heater," Coal Technology, Vol.30, No.07, pp.215-216, July, 2011.

8. Chen Kaikai, "Design of intelligent controller for solar water heater based on single chip microcomputer," Silicon Valley, Vol.7, No.19, pp.9-11, 2014.

9. Luis J. Claros-Marfil, J. Francisco Padial, Benito Lauret, "A new and inexpensive open source data acquisition and controller for solar research: Application to a water-flow glazing," Renewable Energy, Vol.92, pp.450-461, July 2016. 
10. Abbas Zabihi, Majid Taghizadeh, "New energy-saving temperature controller for heater at natural gas gate station, Journal of Natural Gas Science and Engineering, Vol.27, Part 2, pp.10431049, November 2015.

11. Yu Bin, “A Solar Water Heater Controller Based on Single Chip Microcomputer,” Journal of Huzhou Vocational and Technological College, Vol.11, No.1, pp.85-87, 2013.

12. Zhong Huijuan, "The Study of Full Functionalized Controler for Solar Water Heating System Based on FPGA,” Hebei University of Technology, December 2012.

13. Mamdani E H., Application of fuzzy algorithms for control of simple dynamic plant, Proc IEEE, 1974(12).

14. King P J, Mamdani E H., The application of fuzzy control system to industrial processes, Automatic, 1977(5).

15. Huijun Yu, Yong He, Zhengli Zhao, and Min Wu, "Research on Fuzzy PID Pitch-Controlled System Based on SVM," Journal of Advanced Computational Intelligence and Intelligent Informatics, Vol.20, No.2, pp.332-341, 2016.

16. Kazuhiko Terashima, Hideo Kitagawa, Takanori Miyoshi, Sou Kitamura, and Juan Urbano, "Neuro-Fuzzy Control of Power-Assist Omnidirectional Wheelchair Using Human-Friendly Touch Panel,"Journal of Robotics and Mechatronics, Vol.21, No.3, pp.427-434, 2009.

17. Hugang Han and Yuta Higaki, "Controller Designs for a Class of Polynomial Fuzzy Models," Journal of Advanced Computational Intelligence and Intelligent Informatics, Vol.19, No.6, pp.796803, 2015.

18. Zhou Runjing, Zhang Lina, the fuzzy and neural network design based on MATLAB and fuzzy TECH, Electronic Industries Press, 2010.

19. Guo Jia, the application of fuzzy neural network controller based on PLC in the boiler temperature control system in the Hohhot, Inner Mongolia University, 2012.

20. Wang Zhongxian, Modeling and Simulation application of MATLAB, Mechanical Industry Press, 2010. 